DIE ERSTE SEITE

\section{Lebensdauer der Eltern als Indikator für die eigene Mortalität Prognose: steinalt}

Kinder von Eltern, die hochbetagt sterben, erwartet häufig ebenfalls ein langes Leben. Vor Herz- und Gefäßkrankheiten sind sie, einer britischen Studie zufolge, besonders geschützt. Untersucht wurden 186.151 Probanden im Alter von 55 bis 73 Jahren. Es zeigte sich ein umgekehrter Zusammenhang zwischen dem Sterbealter von Mutter oder Vater und der Sterblichkeit der Nachkommen: Die Gesamtmortalität

\section{Ein Kapitel pro Tag}

\section{Leseratten leben länger}

Auch wer keine uralten Eltern hat, muss die Hoffnung auf ein langes Leben nicht aufgeben. $\mathrm{Zu}$ den empfehlenswerten Maßnahmen gehört einer USStudie zufolge auch das Lesen: Senioren, die täglich in Büchern

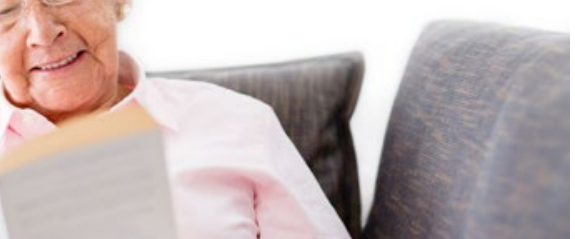

mit jeder Standardabweichung, die das elterliche Sterbealter zunahm, um 9\%, die KHK-Sterblichkeit um $17 \%$ und die Krebssterblichkeit um 8\%. Auch periphere Gefäßerkrankungen, Herzinsuffizienz, Schlaganfall, Hypertonie, KHK, Anämie, Hypercholesterinämie und Vorhofflimmern traten bei Kindern langlebiger Eltern seltener auf.

- JAm Coll Cardiol 2016;68(8):847-875

schmökern, leben durchschnittlich $23 \mathrm{Mo-}$ nate länger als Büchermuffel.

3.635 Personen ab 50 Jahren waren nach ihren Lesegewohnheiten befragt und durchschnittlich 9,5 Jahre lang nachbeobachtet worden. Bücherleser hatten gegenüber Nicht-Lesern einen Überlebensvorteil von $20 \%$. Ein dosisabhängiger Effekt auf die Mortalität zeigte sich bereits, wenn die Probanden mindestens 3,5 Stunden pro Woche mit der Lektüre verbrachten.

- Soc Sci Med 2016;164:44-8

Dr. med. Dirk Einecke Chefredakteur dirk.einecke@springer.com

Prävention von Krebs bis Insult

\title{
Wie viel Bewegung was bringt
}

Wie viel körperliche Aktivität muss man in seinen Alltag einbauen, um sein Risiko für verschiedene Erkrankungen zu senken? US-amerikanische Mediziner haben 174 prospektive Kohortenstudien ausgewertet, 35 zum Mamma-, 19 zum Kolonkarzinom, 55 zum Diabetes, 443 zur KHK und 26 zu ischämischen Insulten.

Im Vergleich zu ungenügend aktiven Menschen wurden für Menschen mit niedrigem (600-3.999 $\mathrm{MET}^{\star}$-Minuten/Woche), mittlerem (4.000-7.999 MET-Minuten) oder hohem Aktivitätsniveau ( $\geq 8.000$ MET-Minuten) folgende Risikoreduktionen ermittelt: Brustkrebs 3\%, 6\% und 14\%; Darmkrebs 10\%, 17\% und 21\%; Diabetes $14 \%, 25 \%$ und $28 \%$; KHK $16 \%, 23 \%$ und $25 \%$; Schlaganfall 16\%, $19 \%$ und $26 \%$.

Um auf die von den Autoren empfohlenen 3.000 MET-Minuten pro Woche zu kommen, eignet sich $\mathrm{z}$. B. folgendes Tagesprogramm: 10 Minuten Treppensteigen, 15 Minuten Staubsaugen, 20 Minuten Gartenarbeit, 20 Minuten Rennen plus $25 \mathrm{Mi}$ nuten Gehen oder Radfahren.

* MET = metabolisches Äquivalent

- BMJ 2016;354:i3857

\section{Langsamer Gang und schwacher Händedruck Dann droht Demenz}

Langsamer Gang und schwacher Händedruck weisen auf ein erhöhtes Demenzrisiko hin. Das geht aus Daten von 2.000 Teilnehmern der Framingham-OffspringStudie hervor. Im Alter von 62 Jahren waren die Probanden ausführlich u.a. mit kognitiven Tests untersucht und anschließend bis zu elf Jahre lang nachbeobachtet worden. Pro Reduktion der Ganggeschwindigkeit um eine Standardabweichung errechnete sich eine Steigerung des
Demenzrisikos um 76\%. Lag die Ganggeschwindigkeit bei maximal $1 \mathrm{~m} / \mathrm{s}$, war das Demenzrisiko fast dreimal so hoch wie bei Probanden, die besser zu Fuß waren. Lag die Stärke des Händedrucks unter dem 10. Perzentil, erhöhte sich das Demenz- bzw. Alzheimerrisiko um den Faktor 2,2-3,2. Langsamer Gang und schwacher Händedruck waren zudem mit einem geringeren Hirnvolumen im MRT assoziiert.

- J Alzheimers Dis 2016;53:1597-1608

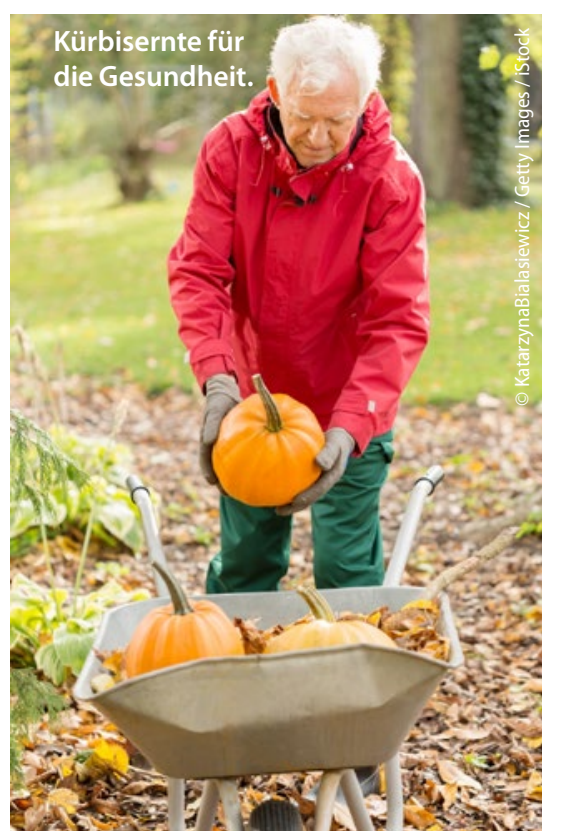

